\title{
A Nonlinear Compound Representation of Sea Clutter
}

\author{
Raghu G. Raj \\ U.S. Naval Research Laboratory, Radar Division \\ Washington D.C. 20375
}

\author{
Alan C. Bovik \\ Dept. of ECE, The University of Texas at Austin \\ Austin, TX 78712
}

\begin{abstract}
We explore the applicability of our recently developed Nonlinear Compound Gaussian (NCG) [1] distribution to modeling the statistics of sea clutter data. We first, for completeness, give a self-contained description of our NCG distribution; both its theoretical properties and the algorithmic details for parameter estimation. We then demonstrate the performance of NCG in modeling the range statistics of seaclutter data [12]. The results clearly demonstrate the superiority of NCG in modeling sea-clutter phenomena over the compound Gaussian (CG) distribution. We conclude with a brief discussion of a phenomenological interpretation of these results together with directions for future research.
\end{abstract}

\section{INTRODUCTION}

Statistical inferences performed in maritime environments entail, in part, the processing of information containing stochastic signatures generated from various physical processes associated with ocean dynamics when sensed by radar systems. We broadly lump together such maritime stochastic phenomena, as measured by a radar, by the term 'sea clutter'. Examples of typical inference operations made by radar systems include the detection and imaging of targets immersed in sea-clutter.

A systematic treatment of such inference processes conducted in maritime environments within a probabilistic framework requires a quantitative description of the stochastic regularities associated with pertinent aspects of sea clutter. A typical example of such a statistical quantity of interest in radar applications is the distribution of sea clutter associated with a given range of interest.

Historically the Gaussian distribution was first used as a crude measure for modeling sea-clutter data. However it became increasingly evident with the advent of highresolution radar that the statistics of sea clutter data deviate significantly from normality [2]. The seminal work of Ward et. al. [2-4] showed that an elegant way to better capture the heavy-tailed structure of sea-clutter data is by the so-called Kdistribution. The $\mathrm{K}$-distribution is a type of compound Gaussian distribution (CG) [5] wherein the $I$ and $Q$ channels of the radar received signal follow a jointly Gaussian random process modulated by a Gamma distribution (it follows, therefore, that the amplitude structure of the combined $I-Q$ data follows a locally Rayleigh distribution whose mean is modulated by a Gamma distributed). The speckle component is usually interpreted to be due primarily to scattering by the capillary waves; while the modulating mean (also called the 'texture') is assumed to be from the ocean gravity waves.

Many researchers have investigated optimal strategies of exploiting this prior knowledge in the service of various radar applications such as detection and imaging [6-7] and significant performance improvement due to such approaches have been reported.

On the other hand there are increasingly many applications and regimes of operation where the $\mathrm{CG}$ distribution fails to satisfactorily model clutter phenomena such as in spiky sea clutter data obtained in high grazing angles [8-9] etc. Moreover different applications have different modeling requirements which the $\mathrm{CG}$ distribution is not able uniformly accommodate due to inherent limitations in its modeling capabilities. For example, in target detection applications it is of greater interest to model the tail portion of the distribution accurately due to the fact that the sea spikes, which are the primary source of interference, is primarily associated with this region; whereas in target imaging applications modeling the entire clutter density is of interest.

We introduce a novel generalization of the CG model called NCG [1] (nonlinear compound Gaussian) distribution which allows us to extend the capabilities of the CG model to better accommodate the variabilities in modeling requirements such as above. We give a self-contained description of this model together with its theoretical properties (in Section 2) and algorithmic details of its parameter estimation (in Section 3 ). Thereafter we present simulation results, in Section 4, demonstrating the superiority of the NCG model in modeling sea-clutter phenomena over the traditional $\mathrm{CG}$ model. We conclude in Section 5 with a brief discussion of some phenomenological interpretations of the NCG model together with directions for future research.

\section{NONILINEAR COMPOUND GAUSSIAN MODEL}

Consider a random variable $X$ that can be decomposed into the following product form (pointwise product of 2 vectors):

$$
X=S \cdot B
$$

The probability density function of $X$ is thus given by:

$$
\begin{aligned}
P_{X}(x) & =\int P_{X}(x \mid B=\beta) P_{B}(\beta) \cdot d \beta \\
& =\int \frac{1}{Z} P_{S}(x / \beta) \cdot P_{B}(\beta) \cdot d \beta
\end{aligned}
$$


where $\mathrm{Z}$ is a normalizing constant for $P_{S}(x / \beta)$.

In the special case where $S \sim N(\mu, \Sigma)$ is a Gaussian random vector with mean $\mu$ and covariance matrix $\Sigma$, and $B$ is a non-Gaussian random vector consisting of i.i.d. components, $X$ in (1) is said to CG distributed random variable:

$$
\begin{array}{r}
P_{X}(x)=\int \frac{1}{\sqrt{2 \pi \mid}|\widetilde{\Sigma}|} \exp \left(-((x-\mu) / \beta)^{H} \tilde{\Sigma}^{-1}((x-\right. \\
\mu) / \beta)) P_{B}(\beta) \cdot d \beta
\end{array}
$$

The NCG model replaces the conditional density $P_{X}(x \mid B=\beta)$ in (2) by a generalized distribution of the following form:

$$
P_{X}(x \mid B=\beta)=\frac{1}{z} g(X) \prod_{i} p_{i}\left(x_{i}\right)
$$

where, $X=\left[x_{1}, \ldots, x_{d}\right]$. The probability densities $p_{i}$ generalize Gaussian random variables in a manner specified below, while the multiplicative term $g(X)$ captures the higher- order statistical interactions between the components of $X$.

We first describe the construction of the NCG distribution in terms of a novel non-linear system model in section II-A. Thereafter in section II-B we present some theoretical properties of the NCG model.

\section{A. Structure of the NCG Distribution}

Let $x=\left[x_{1}, x_{2}, \ldots, x_{d}\right]^{T} \in \mathbb{R}^{d}$ be an observed random vector distributed according to the NCG model (5). We now demonstrate how (5) can be synthesized via the generative model shown in Figure 1. The system F in Figure 1 consists of a core non-linearity $\varphi$ preceded by a linear system $y=A s+$ $\Gamma, \quad$ where $\quad y=\left[y_{1}, y_{2}, \ldots, y_{d}\right]^{T} \in \mathbb{R}^{d} \quad$ and $s=\left[s_{1}, s_{2}, \ldots, s_{d}\right]^{T} \in \mathbb{R}^{d}$ is a Gaussian random vector consisting of i.i.d. components $s_{i} \sim N(0,1)$ whose density is denoted by $q\left(s_{i}\right)$. Vectors $\Gamma=\left[\Gamma_{1}, \Gamma_{2}, \ldots, \Gamma_{d}\right]^{T}$ and $\sigma=$ $\left[\sigma_{1}, \sigma_{2}, \ldots, \sigma_{d}\right]^{T}$ respectively determine the mean and variances for the various Gaussian channels. Matrix $C=\left[\mathrm{C}_{1}, \mathrm{C}_{2}, \ldots, \mathrm{C}_{\mathrm{d}}\right]^{\mathrm{T}}=\mathrm{A}^{-1}$ is assumed to be invertible and thus determines the interaction between the various Gaussian sources. Vector $\beta=\left[\beta_{1}, \beta_{2}, \ldots, \beta_{d}\right]^{T} \in \mathbb{R}^{d}$ is an instantiation of random variable $B$ of the NCG model corresponding to (1). The constants $\gamma_{1}$ and $\gamma_{2}$ play an important role in determining the properties of the non-linear transformation $\varphi$, as we show in the next section. All the operations in system $F$, other than the action of matrix $A$, are pointwise in the components of the vector.

Given this, an important observation is that for a given $B=\beta$, pointwise invertibity of the non-linearity $\varphi$ results in a distribution of the following form (the derivation is straightforward given the above setup):

$$
P_{X}(x \mid B=\beta)=\frac{K}{|J(F)|} g(X) \prod_{k=1}^{d} p_{i}\left(\frac{x_{k}-\mu_{k}}{\beta_{k}}\right)
$$

where $p_{k}\left(\frac{x_{k}-\mu_{k}}{\beta_{k}}\right)=K_{k} \exp \left(-a_{k}\left[\tilde{\varphi}\left(\frac{x_{k}-\mu_{k}}{\beta_{k}}\right)-c_{k}\right]^{2}\right)$

$K_{k}$ is a normalizing constant, $a_{k}=(1 / 2) \sum_{i=1}^{d}\left(C_{i, k}^{2} / \sigma_{k}^{2}\right)$ and $c_{k}=\Gamma_{k}+\frac{\sum_{j \neq k} \sum_{i=1}^{d} \frac{c_{i, j} c_{i, k}}{\sigma_{k}^{2}} \Gamma_{j}}{\sum_{k=1}^{d} \frac{c_{i, k}^{2}}{\sigma_{k}^{2}}}, \tilde{\varphi} \equiv \varphi^{-1}$.

Also, $g(X)=\exp \left[-\sum_{i \neq j} G_{i, j} \tilde{\varphi}\left(\frac{x_{i}-\mu_{i}}{\beta_{i}}\right) \tilde{\varphi}\left(\frac{x_{j}-\mu_{j}}{\beta_{j}}\right)\right]$

where $\quad G_{i, j}=\sum_{k=1}^{d} \frac{C_{k, i} C_{k, j}}{\sigma_{k}^{2}}$ and $K=\frac{\exp \left(-\sum_{k=1}^{d} \frac{\left(C_{k}^{T} \Gamma\right)^{2}}{2 \sigma_{k}^{2}}\right)}{(2 \pi)^{d / 2} \prod_{k=1}^{d} \sigma_{k} K_{k}}$

We have found the following linear-quadratic non-linearity to effectively capture the statistics of real data:

$$
\varphi(s)=\left[\gamma_{1} s \sigma u_{1}(\sigma s)+\gamma_{2} \varphi_{q}(s \sigma) u_{2}(\sigma s)\right] \beta
$$

where, functions $u_{1}$ and $u_{2}$ are defined in the next section, $\varphi_{q}(s)=s^{2} \operatorname{sgn}(s), \beta \geq 0, \sigma \geq 0$, and $s \in \mathbb{R}$.

This non-linearity furnishes a natural generalization of the Gaussian distribution - in particular it consists of both a linear and quadratic channel and reduces to a multidimensional Gaussian distribution when only the linear channel is active.

\section{B. Theoretical Properties of NCG}

As described in the previous section, an important consideration is the characterization of the conditions under which equation (7) is invertible. This is because invertibility makes it possible to generate generalized distributions of the form (5) whereas in general one will obtain a mixture distributions which, though more general, are computationally less tractable. The following Lemma gives a characterization the invertibility of $\varphi$ in (7) for the case where $d=1$ (which therefore applies to the invertibility of the general case since $\varphi$ is a component-wise transform).

Lemma 1. Let $\gamma_{1} u_{1}(s)=\lambda_{1}$ and $\gamma_{2} u_{2}(s)=\lambda_{2}$ such that $\lambda_{1}, \lambda_{2}$ are real constants. Then $\varphi$ in Equation (7) is invertible for all $x$ if and only if $\lambda_{1} \lambda_{2} \geq 0$.

Proof. We consider first the converse case i.e. assume $\lambda_{1} \lambda_{2} \geq 0$

Case 1: $\lambda_{1} \geq 0$ and $\lambda_{2} \geq 0$. Then equation (7) becomes:

$|x|=\left[\gamma_{1}|\sigma||s| u_{1}(\sigma s)+\gamma_{2} \sigma^{2}|s|^{2} u_{2}(\sigma s)\right] \beta \quad$ (since

$\operatorname{sgn}(x)=\operatorname{sgn}(\sigma s))$

$\Rightarrow|s|^{2} \sigma^{2} \beta \lambda_{2}+\sigma \beta \lambda_{1}|s|-|x|=0$

$\Rightarrow|s|=\frac{-\sigma \beta \lambda_{1} \pm \sqrt{\left(\sigma \beta \lambda_{1}\right)^{2}+4|x| \sigma^{2} \beta \lambda_{2}}}{2 \sigma^{2} \beta \lambda_{2}(s)}$

Thus the only feasible solution is:

$$
|s|=\frac{-\sigma \beta \lambda_{1}+\sqrt{\left(\sigma \beta \lambda_{1}\right)^{2}+4|x| \sigma^{2} \beta \lambda_{2}}}{2 \sigma^{2} \beta \lambda_{2}}
$$

Case 2: $\lambda_{1} \leq 0$ and $\lambda_{2} \leq 0$

In this case, $|x| \operatorname{sgn}(x)=-\operatorname{sgn}(s)\left[\gamma_{1}|\sigma||s| u_{1}(\sigma s)+\right.$ $\left.\gamma_{2} \sigma^{2}|s|^{2} u_{2}(\sigma s)\right] \beta$

We once again solve:

$$
|x|=\left[\gamma_{1}|\sigma||s| u_{1}(\sigma s)+\gamma_{2} \sigma^{2}|s|^{2} u_{2}(\sigma s)\right] \beta
$$

to get the unique solution above together with the relationship: $\operatorname{sgn}(x)=-\operatorname{sgn}(\sigma s)$.

Thus in both cases $\varphi$ is invertible thus proving sufficiency.

Now assume that $\varphi$ is invertible. We then have 4 cases: 
Case 1: $\lambda_{1} \geq 0$ and $\lambda_{2} \geq 0$. See above.

Case 2: $\lambda_{1} \leq 0$ and $\lambda_{2} \leq 0$. See above.

For Cases 3 and 4, where Case 3: $\lambda_{1} \geq 0$ and $\lambda_{2}<0$ and Case 4: $\lambda_{1}<0$ and $\lambda_{2} \geq 0$, we are forced to solve the original quadratic problem wherein:

$$
\lambda_{2} \beta \sigma^{2} s^{2} \operatorname{sgn}(s)+\lambda_{1} \beta \sigma s-x=0
$$

It is easy to show for these cases it is always possible to find $x$ such that (8) does not have a unique solution.

From Lemma 1 we note that by setting $\gamma_{1}=1$ and $\gamma_{2}=0$, $N C G$ reduces to the $C G$ distribution as a special case. The following Lemma details a complementary direction in which the above model can be extended.

Lemma 2. Let $\lambda_{1}(s)=\gamma_{1}(s) u_{1}(s)$ and $\lambda_{2}(s)=\gamma_{2}(s) u_{2}(s)$. Then $\varphi$ in (4) is invertible if $\lambda_{1}(s) \lambda_{2}(s)=0$.

Proof. The proof follows by noting that the condition $\lambda_{1}(s) \lambda_{2}(s)=0$ ensures that one and only one of the two channels (i.e. the linear and quadratic channels as described in II-A) are active for a given value of $s$. Thus invertibility of $\varphi$ trivially follows. This establishes the lemma.

We note that under Lemma 2, NCG reduces to the Multilinear ICA (MICA) distribution [10-11] which thus formally complements the CG model. Thus the NCG model subsumes both the CG and MICA distributions as special cases. Importantly the following lemma, furnishes a closed form expression for the Jacobian $|J(X)|$ :

Lemma 3. The Jacobian for the NCG model is:

$$
|J(X)|=|A| \prod_{k=1}^{d} \beta_{k} \psi\left(y_{k}\right)
$$

where $\psi\left(y_{k}\right)=\lambda_{1}(s)+2 \lambda_{2}(s)\left|y_{k}\right|$

$$
\begin{aligned}
& y=\tilde{\varphi}(x) \\
& \lambda_{1}(s)=\gamma_{1}(s) u_{1}(s), \lambda_{2}(s)=\gamma_{2}(s) u_{2}(s)
\end{aligned}
$$

Proof. The proof for the special case of MICA distributions where $\lambda_{1}(s)$ and $\lambda_{2}(s)$ are defined as in Lemma\#2 is given in [10]. Thus we complete the proof for the general case by focusing on the case where $\lambda_{1}(s)=\lambda_{1}$ and $\lambda_{2}(s)=\lambda_{2}$ are constants.

We observe that for all $k, l$ :

$$
\frac{\partial x_{m}}{\partial s_{n}}=A_{m, n} \beta_{m} \psi\left(y_{m}\right)
$$

where,

$$
\psi\left(y_{m}\right)=\lambda_{1}+2 \lambda_{2}\left|y_{m}\right|
$$

Given this we have that:

$$
\begin{aligned}
|J(X)| & =\operatorname{det}\left(\left[\frac{\partial x_{m}}{\partial s_{n}}\right]_{m, n}\right) \\
& =\operatorname{det}\left(\left[A_{m, n} \beta_{m} \psi\left(y_{m}\right)\right]_{m, n}\right) \\
& =\left(\prod_{k=1}^{d} \beta_{k} \psi\left(y_{k}\right)\right) \operatorname{det}(A)
\end{aligned}
$$

Thus the closed form expression for $|J(X)|$ is established for the NCG model.

The existence of the above closed form expression for $|J(X)|$ obviates the need for Monte-Carlo simulation for the estimation of system parameters. The following section delves into more details on the algorithmic aspects of parameter estimation for the NCG model.

\section{NCG PARAMETER EstimAtion AlgORITHM}

We take a two-stage approach to estimating the parameters of the NCG model. Given a set of $N$ pairs of $I-Q$ samples which are organized in a matrix $X \in R^{N x d}$ (alternatively one can also model $\mathrm{N}$ samples of the 1-D amplitude signal $\sqrt{I^{2}+Q^{2}}$ in which case $d=1$ ), we estimate the optimum parameter $\beta$ (assuming the other parameters are known) corresponding to each such sample. Each such pair of $I-Q$ samples is then normalized by the corresponding $\beta$ parameter. Thereafter the optimum NCG parameters are determined in the manner described below. This process is then iterated several times until convergence.

In order to simply matters, we assume as that $B$ is a scalar random variable (note that in general one can have a different $B$ random variable for the $I$ and $Q$ channels; this case can also be handled by the procedure described below). Let $x=$ $\left[x_{1}, \ldots, x_{d}\right]^{T}$ denote a sample from the I-Q pair (i.e. $d=2$ in our case; although this methodology can extend to arbitrary $d$ ). To determine optimum $\beta$ parameter for each I-Q sample we solve the following equation w.r.t. $\beta$ :

$$
\begin{aligned}
& \frac{d P_{X}(x \mid B=\beta)}{d \beta}=\sum_{k=1}^{d} \frac{a_{k}\left|x_{k}\right|}{\lambda_{2} \beta} f\left(\frac{x_{k}}{\beta}\right)+\sum_{i \neq j} G_{i, j}\left[f\left(\frac{x_{i}}{\beta}\right) \operatorname{sgn}\left(x_{j}\right)+\right. \\
& \left.f\left(x_{j} / \beta\right) \operatorname{sgn}\left(x_{i}\right)\right]+\sum_{k=1}^{d} \frac{2 \lambda_{2} \operatorname{sgn}\left(\widetilde{\varphi}\left(x_{k} / \beta\right)\right)}{\lambda_{1}+2 \lambda_{2}\left|\widetilde{\varphi}\left(x_{k} / \beta\right)\right|} \frac{1}{\sqrt{\lambda_{1}^{2}+4 \lambda_{2}\left|x_{k} / \beta\right|}}\left(x_{k} /\right. \\
& \beta)-d=0
\end{aligned}
$$

where, $f(x)=\frac{-\lambda_{1}+\sqrt{\lambda_{1}^{2}+4 \lambda_{2}|x|}}{\sqrt{\lambda_{1}^{2}+4 \lambda_{2}|x|}}$. Since $\beta$ is a scalar parameter, we can easily solve for $\beta$ in a computationally tractable manner by employing a brute force approach of evaluating (10) for every value of $\beta$ in a interval $[0, \max (X)]$ and choosing the $\beta$ that renders the R.H.S. of (10) closest to zero. Furthermore it is easy to verify - both analytically and numerically - that in the $C G$ limit (i.e. $\lambda_{1}=1, \lambda_{2} \rightarrow 0$ ) the optimum solution of (10) is exactly the closed form solution of $\beta$ for the CG case (i.e. $\beta=\sqrt{x^{T} \Sigma^{-1} x / d}$ ) as one would expect.

Having obtained normalized the set of $I-Q$ pairs by random variable $B$, we now detail how to update the remaining parameters of the NCG model. Firstly in our simulations, consistent with the CG model of sea clutter data, we set $\mu=0$ and $\Gamma=0$. Thus the parameters that remain to be estimated are matrix $C$, vector $\sigma$, and the scalars $\lambda_{1}$ and $\lambda_{2}$. For estimating $C$ and $\sigma$, we employ a similar trick as used in [11]: let $y^{i}=\tilde{\varphi}_{\lambda}\left(x^{i} / \beta\right)$ where $x^{i}$ denotes the $i^{\text {th }}$ vector of I-Q coefficients, and where the subscript denotes that the nonlinear function $\tilde{\varphi}$ depends on $\lambda=\left[\lambda_{1}, \lambda_{2}\right]$; then set $\mathrm{C}=\sqrt{\mathrm{Q}}$ ( where $\left.\mathrm{Q}=\frac{1}{N} \sum_{k=1}^{d} y^{k}\left(y^{k}\right)^{\prime}\right)$, and set $\sigma_{i}$ to be the standard deviation of the ith component of the normalized and inverted $I-Q$ coefficients $\left\{y^{k}\right\}_{k}$. Note that the computation of $\mathrm{C}$ and $\sigma$ assumed knowledge of $\lambda$, but did not involve any iterative procedure for estimation.

To compute the optimum $\lambda$ (given knowledge of the other NCG parameters) we resort to an unconstrained iterative maximum-likelihood estimation approach based on the following descent equations: 
$\frac{d P_{X}(x \mid B=\beta)}{d \lambda_{1}}=-\sum_{k=1}^{d} \frac{1}{\psi\left(\widetilde{\varphi}\left(x_{k} / \beta\right)\right)}\left[1+2 \lambda_{2} \operatorname{sgn}\left(\tilde{\varphi}\left(x_{k} /\right.\right.\right.$

$\left.\beta)) \tilde{\varphi}^{\prime}\left(x_{k} / \beta\right)\right]-\sum_{k=1}^{d} 2 a_{k} \tilde{\varphi}\left(x_{k} / \beta\right) \tilde{\varphi}^{\prime}\left(x_{k} / \beta\right)-$

$\sum_{i \neq j} G_{i, j}\left[\tilde{\varphi}\left(x_{i} / \beta\right) \tilde{\varphi}^{\prime}\left(x_{j} / \beta\right)+\tilde{\varphi}\left(x_{j} / \beta\right) \tilde{\varphi}^{\prime}\left(x_{i} / \beta\right)\right]$

A similar gradient equation can also be derived with respect to $\lambda_{2}$. Thus the following algorithm summarizes our NCG parameter estimation procedure:

0) Initialize $\beta$ according to the $\mathrm{CG}$ model. From this initialize $\mathrm{C}$ and $\sigma$.

1) Re-estimate $\beta$ by solving (10)

2) Normalize $X$ according to $\beta$ computed above and reestimate $\mathrm{C}$ and $\sigma$ accordingly

3) Estimate the optimum $\lambda$ via (11). Re-estimate $C$ and $\sigma$ accordingly

Iterate steps (1)-(3) until convergence.

In practice the algorithm converges in a few such iterations. We remark that no a priori texture model has been assumed for the data in the above analysis. Rather the texture model, i.e. the value of $\beta$ on a sample-by-sample basis, is learnt nonparametrically via solving (10). The resulting empirical distribution of $\beta$ can then be analytically approximated via known distributions such as Gamma or inverse-Gamma models. For the IPIX high sea-state sea-clutter data [12] analyzed below the Gamma distribution was found to be a better fit for the data as compared to the inverse-Gamma model.

We note however that intrinsic in the above calculations is the assumption that the texture is uncorrelated on a pulseby-pulse basis. Extensions of this to incorporate fully correlated [13-14] and partially correlated texture models []1516 will be pursued in future work.

\section{ANALYSis of SEA-ClUtTER DATA}

The above NCG parameter estimation algorithm was applied to a sea clutter dataset obtained from [12]. We simultaneously modeled the $I$ and $Q$ channels of the data as described above (similar results were also obtained when modeling the amplitude signal alone). Once we have inferred the parameters of the NCG and CG models corresponding to the data, we generate samples from both of these distributions and match them with the underlying empirical distribution.

Figure 2 shows a snapshot the high sea-state sea clutter dataset described in [12] that we are modeling (we are using the full dataset [12]; Figure 2 shows only a subset of it). Figure 3-a shows the cumulative density functions (cdf's) associated with the empirical, $\mathrm{CG}$ and NCG distributions. We can visually see that the NCG outperforms the CG distribution in modeling the overall distribution; quantitatively, roughly a $30 \%$ decrease in the KLD (Kullback-Leibler Divergence) is achieved by the NCG in modeling the empirical distribution. The NCG distribution thus obtained may be suitable for applications such as imaging. However when examining the tails of the distributions more closely in Figure 3-b we see that in this narrow region the NCG slightly underperforms the $\mathrm{CG}$ model.
However the NCG has considerable flexibility in modeling stochastic phenomena which we exploit as follows. Figure 4-a shows the performance of another NCG distribution when modeling the same dataset, but where $\lambda$ was chosen to better match the tail of the empirical distribution. Apart from outperforming the CG distribution overall, when examining the tail regions of the distributions in Figure 4-b we see that the NCG decisively outperforms CG in the tail region! Thus this type of NCG distribution may be more suitable for target detection applications. Note that we have not optimized the choice of lambda for matching the tail; but have just shown an illustrative example of this. The problem of optimizing $\lambda$ for matching the tail distribution is a subject of future work.

These results therefore illustrate both the flexibility and superiority of the NCG distribution over CG in the modeling of sea clutter phenomena.

\section{DISCUSSION}

We introduced a novel distribution, NCG, that generalizes both the CG and MICA models, and that offers greater flexibility and power in modeling sea clutter statistics - both in modeling the entire distribution and in capturing the tail behavior of the empirical distribution over the traditional $\mathrm{CG}$ model.

We anticipate that this can open up many exciting avenues for various applications of interest in radar signal processing such as in imaging and detection. We further point out in this connection that since $\mathrm{CG}$ model has been tremendously useful in image processing for applications such as denoising, we should expect similar applicability of the NCG model beyond the modeling of sea-clutter [1]. Thus NCG is a general model that could be used a building block for more complex probabilistic models of both sea-clutter and scene structure.

Turning now to the phenomenological underpinnings of the NCG model in relation to sea clutter, we speculate that the quadratic non-linearity that we have introduced in the NCG model is related to the coupling between the capillary and gravity waves which may manifest itself in terms of higher order correlations in the multiplicative term $g(X)$. The exploration of these and related questions stemming from this work is a subject of future work.

\section{REFERENCES}

[1] R.G. Raj and A.C. Bovik, "The Multilinear Compound Gaussian Distribution" International Conf. on Acoustics, Speech and Signal Processing (ICASSP), Kyoto, March 2012.

[2] K.D. Ward, "Compound representation of high-resolution sea-clutter," Electronics Letters, 17(16), pp. 561-563, 1981.

[3] S. Watts and K.D. Ward, "Spatial Correlation in K-Distributed Sea Clutter," Proc. IEE Part F, 134(6), pp. 526-532, 1987.

[4] K.D. Ward, R.J.A. Tough and S. Watts, Sea Clutter: Scattering, the KDistribution and Radar Performance, IET Radar, Sonar and Navigation Series 20, 2006.

[5] J. Wang, A. Dogandzic, and A. Nehorai,"Maximum likelihood estimation of compound-gaussian clutter and target parameters," IEEE Trans. Signal Processing, vol. 54, no. 10, October 2006.

[6] K.J. Sangston, F. Gini, M.V. Greco, and A. Farina, "Structures for radar detection in compound gaussian clutter," IEEE Transactions in Aerospace and Electronics Systems, vol. 35, no. 2, April 1999. 
[7] F. Gini, A. Farina, and F. Lombardini, "Effects of Foliage on the Formation of K-Distributed SAR Imagery," Signal Processing, vol. 75, No. 2, pp. 161-172, June 1999.

[8] Y. Dong, "Distribution of X-Band High Resolution and High Grazing Angle Sea Clutter," Technical Report, DSTO-RR-0136, Defense Science Technology Organization, Australia, 2006.

[9] Y. Dong, "High Grazing Angle and High Resolution Sea Clutter: Correlation and Polarisation Analyses," Technical Report, DSTO-TR1972, Defense Science Technology Organization, Australia, 2007.

[10] R.G. Raj and A.C. Bovik, "MICA: A multilinear ICA decomposition for natural image modeling," IEEE Trans. on Image Processing, vol: 17, no: 3, March 2008, Page(s): 259-271.

[11] R.G. Raj and A.C. Bovik, "A fast multilinear ICA Algorithm," IEEE International Conference on Image Processing, Hong Kong 2010.

[12] IPIX Radar Database:

http://soma.crl.mcmaster.ca/ipix/dartmouth/datasets.html
[13] A. Farina, F. Gini, M.V. Greco, and L. Verrazzani, "High resolution sea clutter data: statistical analysis of recorded live data," IEE Proceedings - Radar, Sonar and Navigation, vol. 144, no. 3, pp.121-130, 1997.

[14] K.J. Sangston, F. Gini, M.S. Greco, "New results on coherent radar target detection in heavy-tailed compound-Gaussian clutter," IEEE Radar Conference, Washington D.C. 2010.

[15] F. Gini and M. Greco,"Texture modelling, estimation and validation using measured sea clutter data," IEE Proceedings - Radar, Sonar and Navigation, vol. 149, no. 3, pp.115 - 124, 2002.

[16] M. Greco, F. Bordoni, F. Gini,"X-band sea-clutter nonstationarity: influence of long waves," IEEE Journal of Oceanic Engineering, vol. 29, no. 2, pp.269-283, 2004.

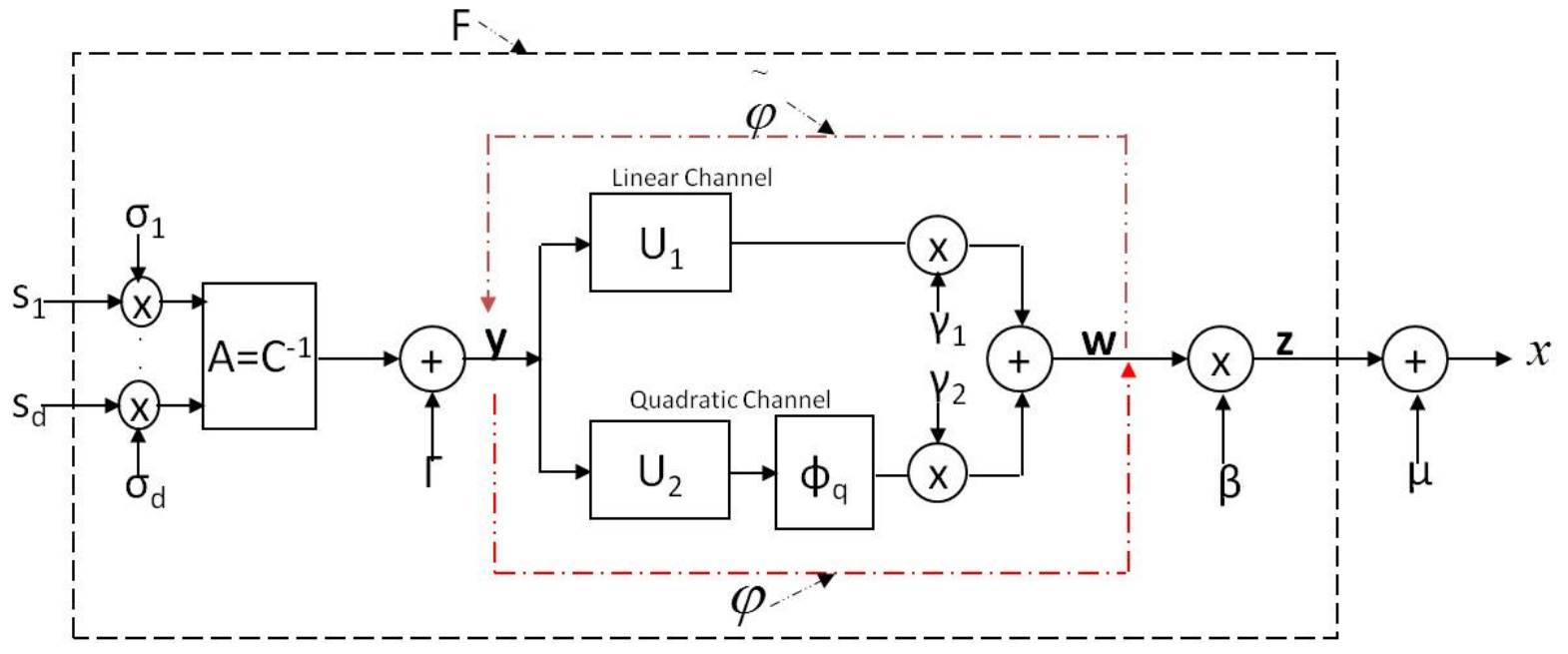

Figure 1
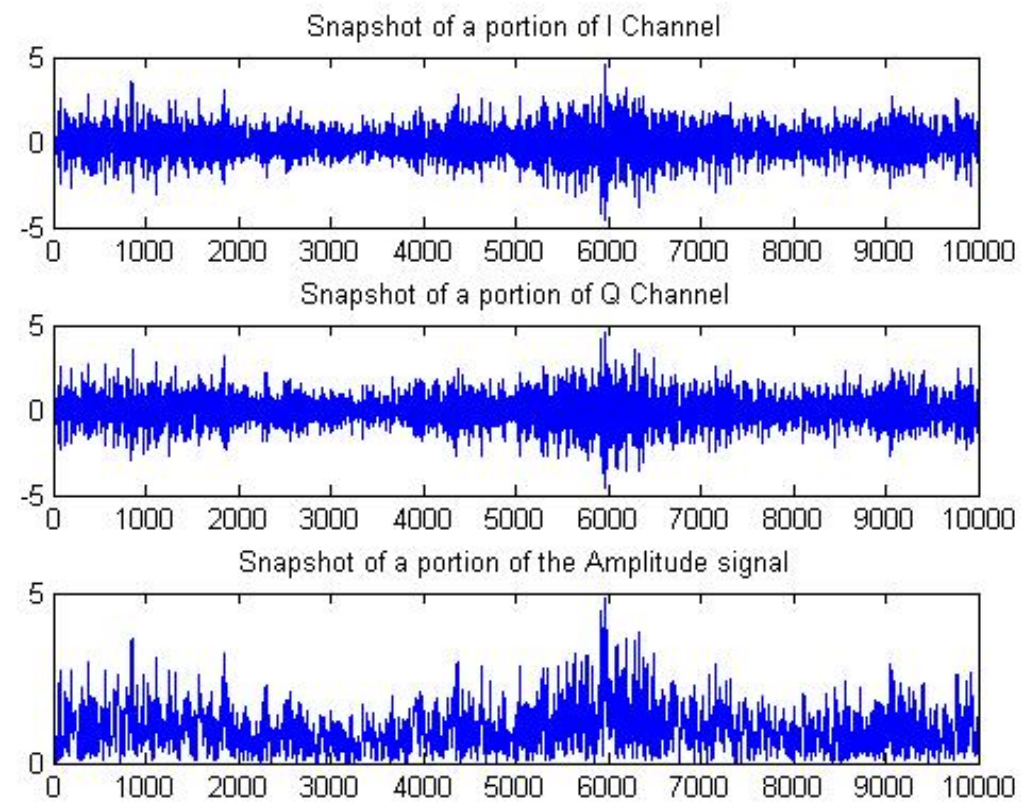

Figure 2: A Snapshot of a portion of the High Sea-state Sea-Clutter Data [12] 


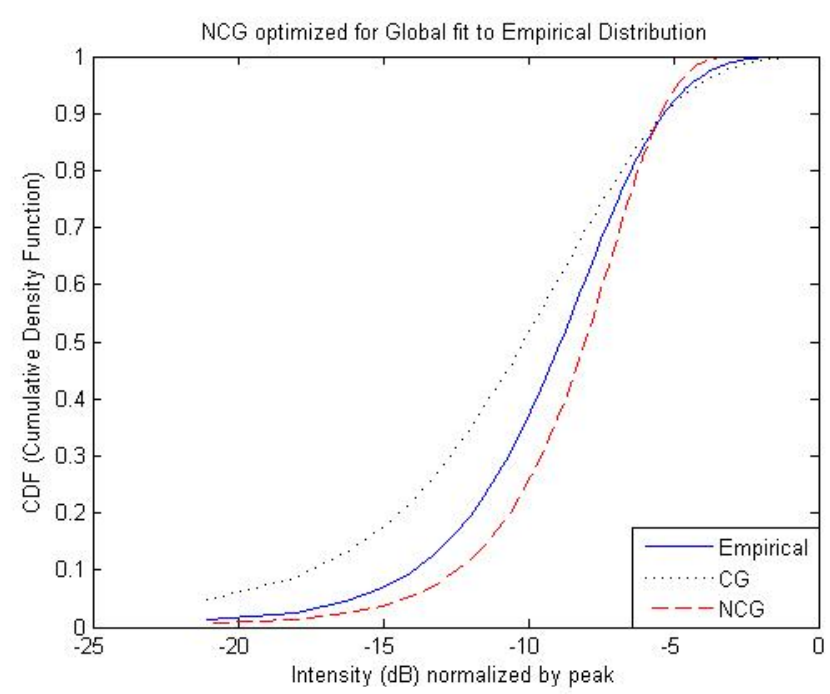

Fig. 3-a

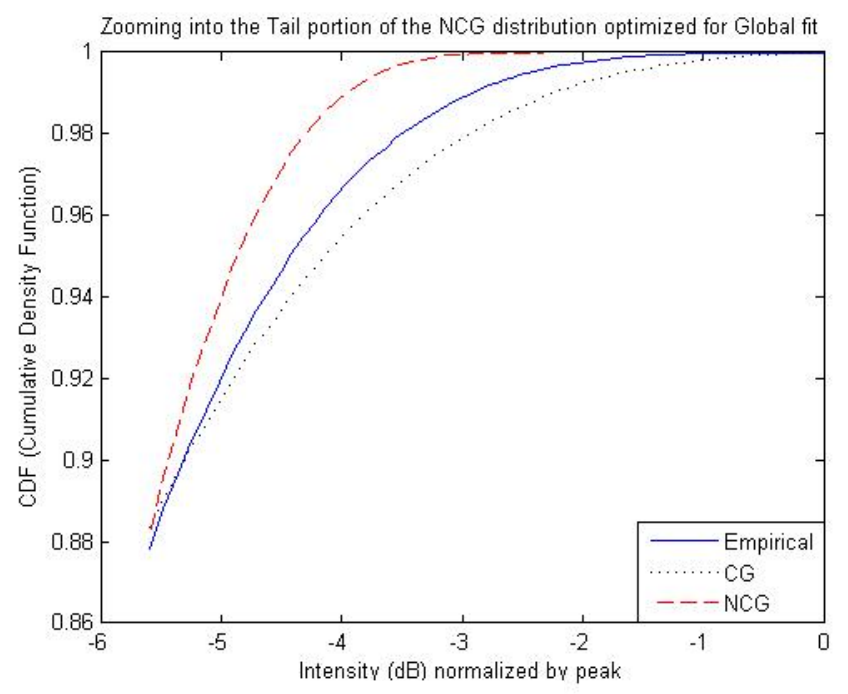

Fig. 3-b

Figure 3-a: NCG Distribution Optimized for Global fit of the Empirical distribution. The full cdf is shown here. Quantitatively NCG outperforms CG in terms of Global fit to the Empirical distribution.

Figure 3-b: The same NCG Distribution Optimized as in Figure 3-a - but just zoomed into the tail. Note that the tail performance of the NCG is not as good as that of the CG (since it is not optimized for the tail); this is addressed in Figure 4

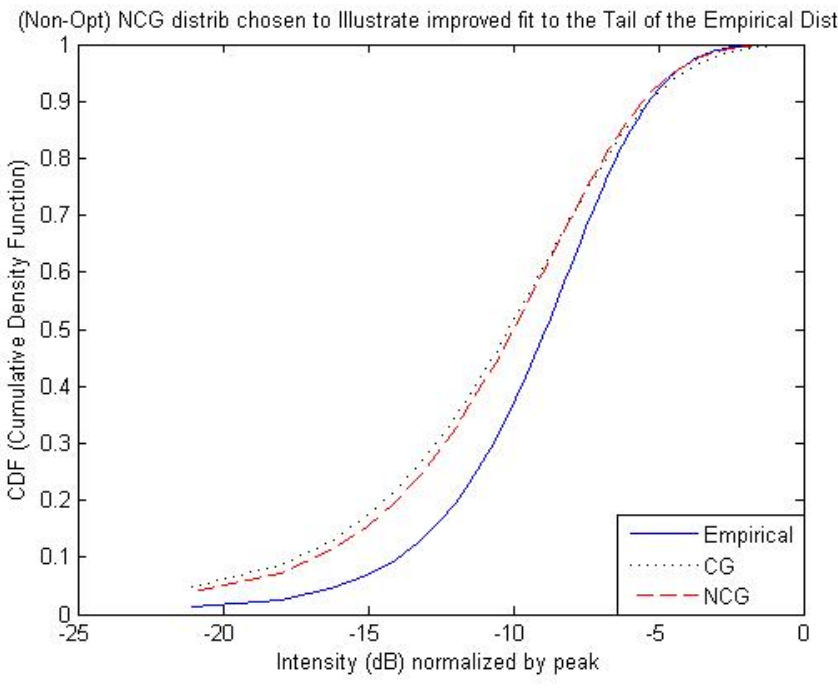

Fig. 4-a

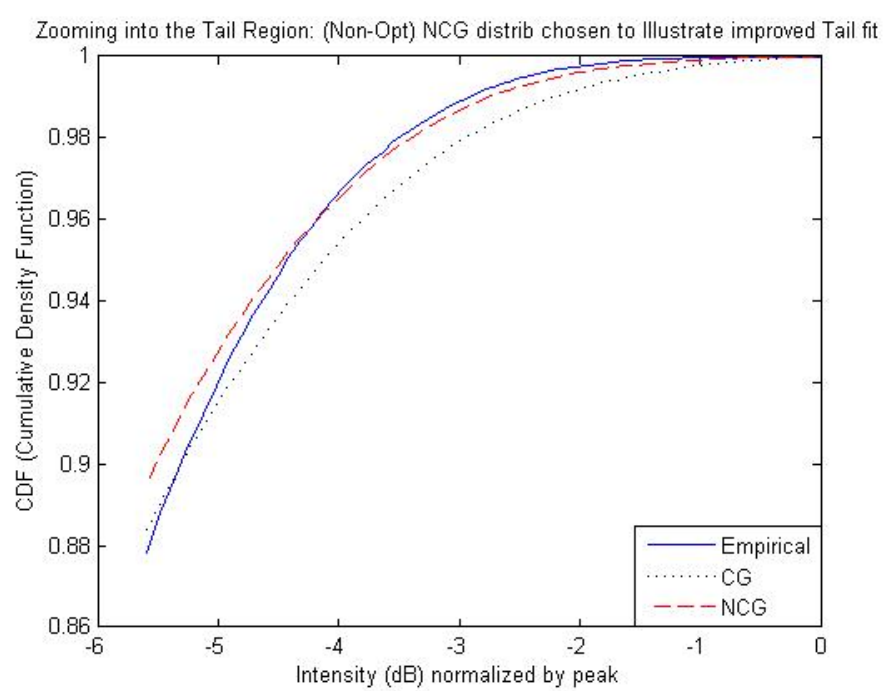

Fig. 4-b

Figure 4-a: NCG Distribution Chosen to better fit the tail of the Empirical distribution. The full cdf is shown here. Here NCG quantitatively outperforms CG slightly in terms of Global fit to the Empirical distribution; but also significantly in terms of Tail fit

Figure 4-b: The same NCG Distribution Optimized as in Figure 4-a - but just zoomed into the tail.

Note that the NCG significantly outperforms CG in the tail region. 